\title{
Evidence for the existence of localized plastic flow auto-waves generated in deforming metals
}

\author{
Lev B. Zuev*, Svetlana A. Barannikova \\ ${ }^{1}$ Institute of Strength Physics and Materials Science, Siberian Branch of Russian Academy of Sciences, Tomsk, Russia; \\ bsa@ispms.tsc.ru; ; Corresponding Author: $\underline{\text { lbz@ispms.tsc.ru }}$
}

Received 12 January 2009; revised 26 February 2009; accepted 8 March 2009.

\begin{abstract}
The localized plastic flow auto-waves observed for the stages of easy glide and linear work hardening in a number of metals are considered. The propagation rates were determined experimentally for the auto-waves in question with the aid of focused-image holography. The dispersion relation of quadratic form derived for localized plastic flow auto-waves and the dependencies of phase and group rates on wave number are discussed. A detailed comparison of the quantitative characteristics of phase and group waves has revealed that the two types of wave are closely related. An invariant is introduced for localized plastic flow phenomena occurring on the micro-and macro-scale levels in the deforming solid.
\end{abstract}

Keywords: Metallic Materials; Mechanical Testing; Optical Interferometry; Strengthening and Mechanisms; Crystal Plasticity; Fracture

\section{INTRODUCTION}

The experimental evidence obtained previously [1-4] suggests that the plastic deformation tends to localize in the deforming solid over the entire flow process. Plastic flow localization is most pronounced on the macro-scale level where the type of local strain pattern is governed by the law of work hardening acting at a given flow stage, i.e. $\theta \equiv E^{-1} \cdot d \sigma / d \varepsilon=\theta(\varepsilon)$ (here $E$ is the elasticity modulus). The localization of plastic deformation will assume in this case the form of auto-wave ${ }^{1}$, i.e. a

\footnotetext{
${ }^{1}$ Autowaves are opposed to, e.g. elastic waves of the type $\sin (w t-k x)$, in that they are solutions to parabolic differential equations in the partial derivatives $\dot{y}=\phi(x, y)+D y^{\prime \prime}[5]$, while the latter obey hyperbolic equations of the type $\ddot{y}=c^{2} \cdot y^{\prime \prime}$.
}

self-excited process $[3,4]$. The occurrence of autowave processes by the plastic deformation is considered, e.g. in the context of gradient plasticity theory [6-8].

A considerable body of experimental and theoretical evidence pertaining to plastic flow macro-localization has been obtained thus far $[1-4,6]$, which suggests that the macro-scale inhomogeneities of localized plastic flow have a typical scale of about $10^{-2} \mathrm{~m}$. A characteristic picture is created in the deforming specimen where deformed material zones move in a concerted manner, generating thereby localized plastic flow auto-waves, which have typical wavelength of about $10^{-2} \mathrm{~m}$. Thus a deforming body would spontaneously separate into alternating deformed and undeformed zones (Figure 1). Following H. Hacken [9], the spontaneous emergence of plastic flow inhomogeneities might be regarded as a manifestation of self-organization processes occurring in the deforming medium.

\section{EXPERIMENTAL PROCEDURE AND MATERIALS TESTED}

On the base of available experimental evidence [1-4], the quantitative characteristics of auto-wave processes were determined for a wide circle of pure metals and alloys, both single crystals and polycrystalline ones, having FCC, BCC and HCP crystal lattice. It is found that the mechanical characteristics of investigated materials and the shape of plastic flow curves obtained for the test specimens would vary significantly, depending on chemical composition, grain size of polycrystalline materials and extension axis orientation of single crystals. In what follows the distinctive features common to all the investigated materials are discussed.

The experimental observations of localized plastic flow auto-waves [1-4] were conducted using the technique of focused-image holography related to speckle photography [10]. This technique was specially deve- 
loped to facilitate the determination of displacement vector fields and the calculation of plastic distortion tensor components for the deforming specimen. One can visualize localized plastic flow nuclei, using the spatial distributions of plastic distortion tensor components; the kinetics of nuclei motion can be determined from the temporal evolution of nuclei.

The most interesting scenario is realized in single crystals and polycrystalline specimens tested in tension at a constant rate.

At the stages of easy glide and linear work hardening localized plastic flow auto-waves would be generated in the deforming specimen where the flow stress $\sigma$ is related to the deformation $\varepsilon$ as $\sigma \sim \theta_{i} \varepsilon$ (here $i=1,2$ for easy glide stage and linear work hardening, respectively, and $\left.\theta_{1} \ll \theta_{2}\right)$. The emergent picture comprises a set of equidistant localization zones, which moves as a whole at a constant rate, generating thereby so-called phase auto-waves (Figure 1). The nature of phase auto-waves merits special study.

The main characteristics of auto-waves, i.e. wavelength $\lambda$ and period $T$, are determined from the co-ordinates of nuclei against time (see Figure 2). Then the propagation rate of auto-waves is estimated as $V_{a w}=\lambda / T=\omega / k \quad$ (here $\omega=2 \pi / T$ is the frequency and $k=2 \pi / \lambda$, the wave number).

1) auto-wave propagation rate;

2) dispersion relation for auto-waves;

3) change in the entropy of the system upon autowave generation;

4) correspondence between the emergent pattern and the given flow stage.

\section{CHARACTERISTICS OF LOCALIZED PLASTIC FLOW AUTOWAVES: EXPERIMENTAL RESULTS}

\subsection{Autowave Rate}

Our findings $[1,2]$ and complementary information along these lines obtained by other workers permit the following conclusion: the propagation rate of auto-waves is a function of the work hardening coefficient, i.e.

$$
\mathrm{V}_{\mathrm{aw}}=\mathrm{V}_{0}+\Xi / \theta \sim 1 / \theta
$$

where $V_{0}$ and $\Xi$ are constants and $V_{0}<<\Xi$. It is of importance that auto-wave rates are in the range $10^{-5} \leq V_{a w} \leq 10^{-4} \mathrm{~m} \cdot \mathrm{s}^{-1}$. Relation (1) applies to both the easy glide and the linear work hardening stage, with the constants $V_{0}$ and $\Xi$ having different values for the same stages.

To begin our discussion of the nature of auto-wave processes, we must mention that plasticity waves occurring by impact loading are described in sufficient detail (see, e.g. [11]).

Plasticity wave rates are in the range $10 \leq V_{p w}$ $=(\theta / \rho)^{1 / 2} \leq 10^{2} \mathrm{~m} \cdot \mathrm{s}^{-1}\left(\right.$ cf. $\left.10^{-5} \leq V_{a w} \leq 10^{-4} \mathrm{~m} \cdot \mathrm{s}^{-1}\right)$ Apparently, $V_{a w}<<V_{p w}$.

Besides, the dependencies of wave rate on work hardening coefficient, $V(\theta)$, obtained for these two types of wave differ essentially in form, i.e. $V_{p w} \sim \varepsilon^{1 / 2}$ [11]) and $V_{a w} \sim \theta^{-1}$ (see Eq.1). The latter relation holds good for all the investigated materials whose plastic flow curve shows easy glide and linear work hardening stages. Thus, the above quantitative analysis of the wave characteristics suggests that we are dealing here with two altogether different types of wave.

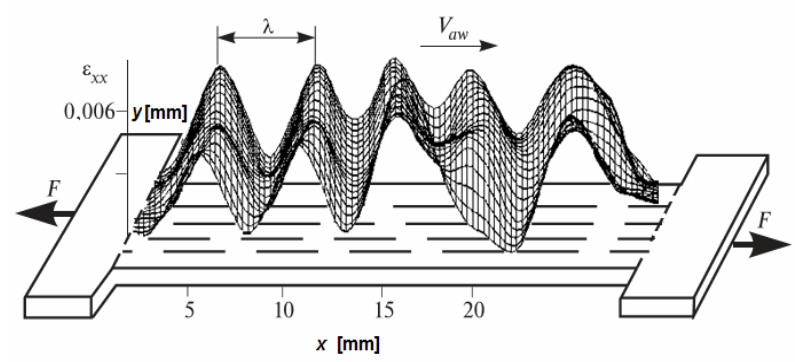

Figure 1. Auto-wave of plastic deformation localization propagating at the linear work hardening stage in the tensile single crystal of alloyed $\mathrm{Fe} ; \varepsilon_{x x}$-local elongation; $x$ and $y$-specimen length and width, respectively; $F$-external load; $\lambda$-spacing of nuclei (auto-wave length); $V_{a w}$-auto-wave propagation rate.

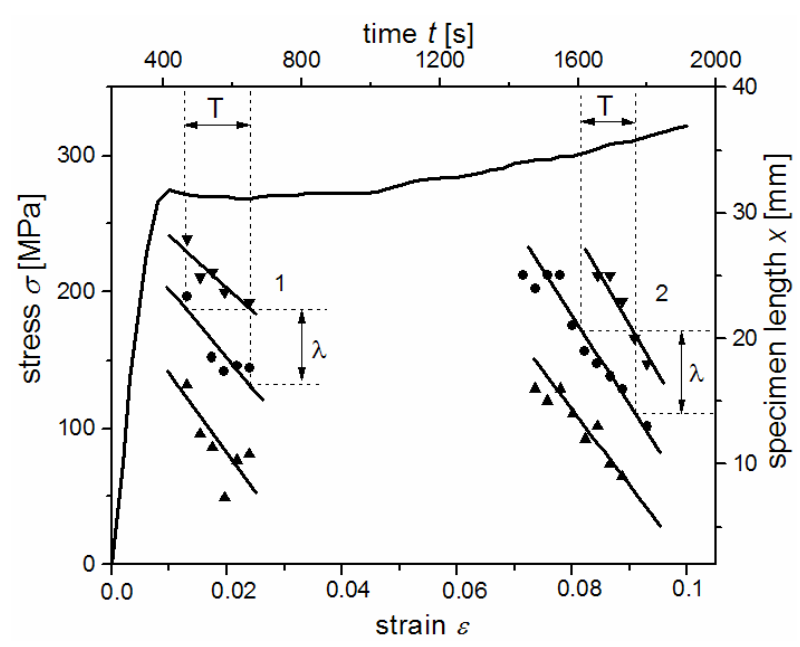

Figure 2. Determination of the spatial $(\lambda)$ and temporal $(T)$ periods of localized plastic deformation for the stages of easy glide (1) and linear work hardening (2) in single crystals of alloyed $\mathrm{Fe} ; \sigma(\varepsilon)$-stress-strain dependence; $X(t)$-variation in the co-ordinates $(\boldsymbol{\Lambda}, \bullet$ and $\boldsymbol{\nabla})$ of localization nuclei with time. 


\subsection{Dispersion Relation for Autowaves}

To gain a better understanding of the nature of autowave processes involved in plastic flow localization, one must consider the dispersion relation $\omega(k)$, which is characteristic for localized plastic flow auto-waves generated at linear work hardening stage [12].

Relation $\omega(k)$ has been complemented by an additional branch, which corresponds to the occurrence of a periodic localization pattern at the easy glide stage (see Figure 3(a)), i.e.

$$
\omega(k)=\omega_{0} \pm \alpha\left(k-k_{0}\right)^{2}
$$

where, $\alpha, \omega_{0}$ and $k_{0}$ are constants, which depend on work hardening stage and kind of material. Note that for easy glide, $\alpha<0$ and for linear work hardening, $\alpha>0$.Substituting into relation (2) of $\omega=\omega_{0} \cdot \widetilde{\omega}$ and $k=k_{0}+\tilde{k} \cdot\left(\operatorname{sign} \alpha \cdot \alpha / \omega_{0}\right)^{-1 / 2}$ (here $\widetilde{\omega}$ is the dimensionless frequency and $\tilde{k}$ is the wave number and $\operatorname{sign} \alpha=+1$ for $\alpha>0$; sign $\alpha=-1$ for $\alpha<0$ is a signum function of the term from Eq.2) yields the following canonic formula $\tilde{\omega}=1 \pm \tilde{k}^{2}$ (see the plot presented in Figure 3(b)).

The above dispersion relation of quadratic form satisfies the Schrödinger nonlinear equation [13,14] commonly applied to self-organization processes occurring in active nonlinear media, which is an undeniable proof that plastic flow localization is a process involved in the self-organization of the deforming medium.

\section{DISCUSSION OF RESULTS}

\subsection{Invariant for Deformation Processes}

On the base of experimental data a close correlation has been established between the product of auto-wave macroscopic parameters, $\lambda \cdot V_{a w}$, and that of material microscopic parameters, $d \cdot V_{\perp}$ (here $d$ is the spacing between close-packed planes of the lattice and $V_{\perp}$ is the rate of transverse elastic waves).

The Table 1 lists numerical data for seven metals investigated. In each instance, the following equality apparently holds good within an acceptable range of accuracy, i.e.

$$
\lambda \cdot V_{a w} \approx 1 / 2 d \cdot V_{\perp}
$$

where the terms have the units of the diffusion coefficient $\mathrm{L}^{2} \cdot \mathrm{T}^{-1}$. To verify relation (3), we used borrowed values of $d$ and $V_{\perp}[15,16]$. Relation (3) was averaged for easy glide and linear work hardening stages as $\left\langle 2 \lambda \cdot \mathrm{V}_{\mathrm{aw}} / \mathrm{d} \cdot \mathrm{V}_{\perp}\right\rangle \approx(1.04 \pm 0.14) \approx 1 . \mathbf{E q} .3$ was plotted in the dimensionless co-ordinates $\lambda / \mathrm{d}-\mathrm{V}_{\perp} / \mathrm{V}_{\text {aw }}$ to give a rectilinear diagram $\lambda / \mathrm{d}=0.82 \cdot 10^{7}+0.22 \cdot \mathrm{V}_{\perp} / \mathrm{V}_{\mathrm{aw}}$ (Figure 4).

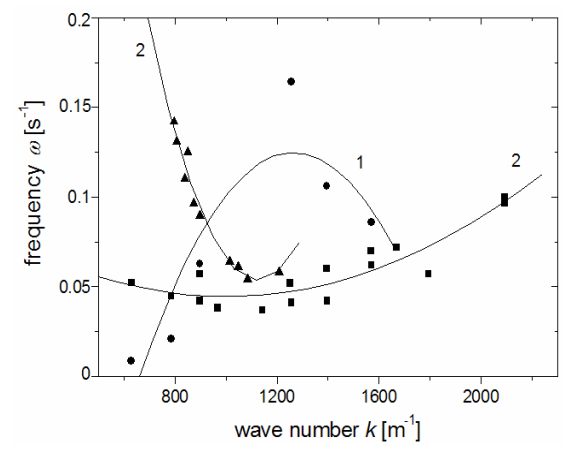

(a)

- - single crystals of $\mathrm{Cu}, \mathrm{Sn}$ and alloyed $\mathrm{Fe}$;

- single crystals of alloyed $\mathrm{Fe}$;

$\Delta$ - polycrystalline $\mathrm{Al}$;

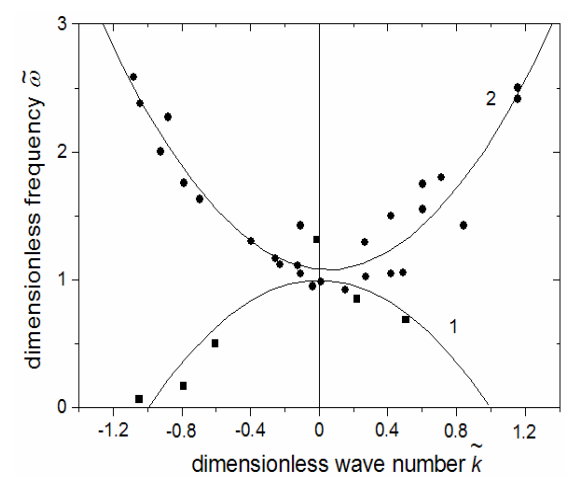

(b)

- - easy glide stage;

- - linear work hardening stage.

Figure 3. A generalized dispersion curve obtained for the stages of easy glide. (1) and linear work hardening (2) in the test specimens; (a) -original data $\omega(k)$; (b) -canonical form of dispersion relation in the dimensionless variables $\tilde{\omega}(\tilde{k})$.

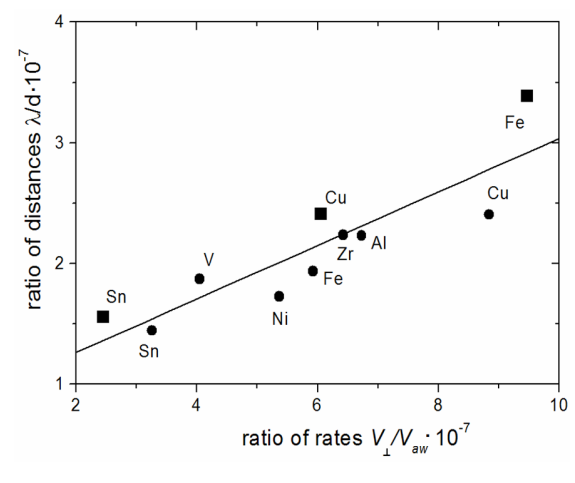

- - easy glide stage; $\bullet$ - linear work hardening stage.

Figure 4. Verification of the validity of relation (2) plotted for auto-waves in the dimensionless co-ordinates $\lambda / d-V_{\perp} / V_{a w}$. 
Table 1. The products $\lambda \cdot V_{a w}$ and $d \cdot V_{\perp}$ matched for easy glide and linear work hardening stages.

\begin{tabular}{|c|c|c|c|c|c|c|c|}
\hline Flow stage & Metal & $\lambda \cdot V_{a w} \times 10^{7}\left(\mathrm{~m}^{2} / \mathrm{s}\right)$ & $D \times 10^{10}(\mathrm{~m})$ & $V_{\perp}$ & $\times 10^{-3}(\mathrm{~m} / \mathrm{s})$ & $d \cdot V_{\perp} \times 10^{7}\left(\mathrm{~m}^{2} / \mathrm{s}\right)$ & $\frac{2 \lambda \cdot V_{a w}}{d \cdot V_{\perp}}$ \\
\hline \multirow{4}{*}{ Easy glide } & $\mathrm{Cu}$ & 1.90 & 2.08 & & 2.30 & 4.78 & 0.79 \\
\hline & $\mathrm{Fe}$ & 3.03 & 2.07 & & 3.32 & 6.87 & 0.88 \\
\hline & Sn & 3.28 & 2.91 & & 1.79 & 5.20 & 1.26 \\
\hline & $\mathrm{Cu}$ & 3.60 & 2.08 & & 2.30 & 4.78 & 1.50 \\
\hline \multirow{6}{*}{$\begin{array}{l}\text { Linear work } \\
\text { harde-ning }\end{array}$} & $\mathrm{Al}$ & 7.92 & 2.33 & & 3.23 & 7.52 & 2.10 \\
\hline & $\mathrm{Zr}$ & 1.92 & 2.46 & & 2.25 & 5.53 & 0.70 \\
\hline & $\mathrm{V}$ & 2.80 & 2.14 & & 2.83 & 6.06 & 0.92 \\
\hline & $\mathrm{Fe}$ & 2.55 & 2.07 & & 3.32 & 6.87 & 0.74 \\
\hline & $\mathrm{Ni}$ & 2.10 & 2.03 & & 3.22 & 6.54 & 0.64 \\
\hline & Sn & 2.34 & 2.91 & & 1.79 & 5.20 & 0.90 \\
\hline
\end{tabular}

Eq.3 relates the micro-scale characteristics $\mathrm{d}$ and $V_{\perp}$, which are observed for elastic waves propa-gating in crystals, to the macro-scale parameters $\lambda$ and $V_{a w}$, which are observed for elastic waves propagating in crystals, to the macro-scale parameters $\lambda$ and $V_{a w}$, which are obtained for localized plastic flow auto-waves generated in the same crystals. The products of these values, $d \cdot V_{\perp}$ and $\lambda \cdot V_{a w}$, are invariants for elastic and plastic deformation processes, respectively $(<<1$ and $\approx 1$, respectively). The above regularity stems from the fact that the processes of elastic and plastic deformation are closely related. In the course of deformation the redistribution of elastic stresses occurs via micro-scale processes at the rate $V_{\perp}$, while the rearrangement of localized plastic flow nuclei involves macro-scale processes occurring at the rate $V_{a w}$, with the processes of both types being related by Eq.3. Thus, the macrolocalization phenomena must be regarded as an attribute of plastic deformation rather than a random disturbance of plastic flow homogeneity.

\subsection{On the Physical Meaning of Relation (1)}

By considering the nature of localized plastic flow auto-waves, it might be pertinent to discuss the origin of dependency $V_{a w} \sim \theta^{-1}$ and, in particular, the meaning of deformation processes occurring in crystals is the propagation rate of elastic waves, i.e. sound rate $V_{S}$. For most metals $V_{S} \approx 5 \cdot 10^{3} \mathrm{~m} \cdot \mathrm{s}^{-1}$; hence $V_{S} / \Xi \approx 10^{10}$. To account for relation (1), the Dirac large-numbers hypothesis [17] was invoked. An appropriate dimensionless relation of the same order was also required which could be applied to the quantities associated with the deformation processes ${ }^{2}$. The relation of deforming medium's viscosities defined for two limiting cases was thought to be an appropriate one, with the limiting cases being the quasi-viscous motion of dislocations, involving no interaction with local obstacles, and the breakaway of dislocation segments from local obstacles. In the former case, the motion of dislocations occurs at high acting stresses; the dislocation velocity as a function of applied stress $\sigma$ has the form $V_{\text {disl }}=b \sigma / B$ (here $b$ is the Bürgers vector; $\mathrm{B} \approx(1-3) \cdot 10^{-4} \mathrm{~Pa} \cdot \mathrm{s}$ is the coefficient of dislocation drag, which characterizes the viscosity of phonon gas in the crystal) [18]. In the latter case, viscosity is defined from internal friction measurements to yield $\eta \geq 3 \cdot 10^{6} \mathrm{~Pa} \cdot \mathrm{s}$ [19]. In the case of ultrasound waves, stresses have low amplitudes; therefore, the viscosities observed for micro-scale plastic deformation processes might have similar values. Hence the ratio $\eta / \mathrm{B} \approx 10^{10}$ and, consequently, $\mathrm{V}_{\mathrm{S}} / \Xi \approx \eta / \mathrm{B}$. Then one can write

$$
\Xi \approx \mathrm{B} / \eta \cdot \mathrm{V}_{\mathrm{S}}
$$

Eq.4 can be interpreted as follows. Complex systems capable of structure formation will spontaneously separate into an information subsystem and a dynamic one [20]. It is thus assumed that the information subsystem, which is characterized by low-amplitude stresses and high viscosity, is represented by acoustic emission signals whereas the dynamic subsystem, which is characterized by high-amplitude stresses and low viscosity, is represented by the motion of individual dislocations and dislocation ensembles, with Eq.4 formalizing the relationship between the two subsystems. Thus, the former subsystem is related to the processes involving elastic wave propagation and the latter, to dislocation plasticity proper.

To better understand the physical meaning of Eq.1, one can also invoke the notions incorporated into the concept of work hardening. It is assumed that $d V_{a w} / d \theta \sim l(\varepsilon)$ (here 1 is the length of slip line). According to the work hardening concept proposed by Seeger [21], $l(\varepsilon)=\Lambda /\left(\varepsilon-\varepsilon^{*}\right)$, with and $\varepsilon^{*}$ depending on material kind; for linear work hardening, the coeffi- cient of work hardening $\theta \approx(n b / 3 \Lambda)^{1 / 2}$ (here $b$ is the Bürgers vector of dislocations and $\mathrm{n}$ is the number of dislocations in a dislocation pileup). In the latter case, $\Lambda \sim \theta^{-2}$, i.e. 


$$
\mathrm{dV}_{\mathrm{aw}} \approx \mathrm{\kappa} \cdot \mathrm{nb} \cdot 1 /\left(\varepsilon-\varepsilon^{*}\right) \cdot \mathrm{d} \theta / \theta^{2}
$$

where the coefficient has the units $\left[\mathrm{T}^{-1}\right]$. With $\varepsilon-\varepsilon^{*}$ depending only weakly on material type [21], Eq.1 can be derived from (5), considering that

$$
V_{a w} \approx \kappa \cdot n b /\left(\varepsilon^{*}-\varepsilon\right) \cdot 1 / \theta
$$

where $\kappa \cdot n b /\left(\varepsilon^{*}-\varepsilon\right) \equiv \Xi$.

The coefficient $\Xi$ can be calculated by taking into account that the values and $\varepsilon^{*}$ depend only weakly on material kind and deformation [21]. Indeed, the derivative $d V_{a w} / d \theta \approx 1.5 \cdot 10^{-2} \mathrm{~m} \cdot \mathrm{s}$ can be estimated from the data reported in $[1,2]$. For the increment in the deformation $\delta \varepsilon \approx 0.05$, an increase in the length of slip line $l$ is about $3 \cdot 10^{-4} \mathrm{~m}$ [21]. Hence $\kappa \approx\left(\mathrm{dV}_{\mathrm{aw}} / \mathrm{d} \theta\right) / \delta \varepsilon \approx 10$ $\mathrm{s}^{-1}$. Provided $n \approx 20[21]$ and $b \approx 2 \cdot 10^{-10} \mathrm{~m}, \Xi \approx 8 \cdot 10^{-7}$ $\mathrm{m} \cdot \mathrm{s}^{-1}$, which is close to the experimental value $\Xi \approx$ $5 \cdot 10^{-7} \mathrm{~m} \cdot \mathrm{s}^{-1}$.

The physical significance of the above difference lies in the fact that localized plasticflow auto-waves belong to an altogether different class of wave phenomena, which are not identical with plasticity waves [11]. Therefore, these two types of waves cannot be grouped together.

\subsection{Treatment of Dispersion Relation}

Dispersion relation (2) of quadratic form can be explained as follows. Relation (3) can be rewritten as

$$
V_{a w}=\left(d \cdot V_{\perp} / 2\right) \cdot 1 / \lambda=\left(d \cdot V_{\perp} / 4 \pi\right) \cdot k=\varsigma \cdot k
$$

Let the rate of localized plastic flow auto-wave, $V_{a w}=V_{g r}$ (here $\mathrm{V}_{\mathrm{gr}}$ is the group rate); then $V_{a w}=d \omega / d k$. Hence

$$
d \omega=\varsigma \cdot k \cdot d k
$$

Integration can be performed for Eq.8 as follows

$$
\int_{\omega_{0}}^{\omega} d \omega=\varsigma \int_{0}^{k-k_{0}} k \cdot d k
$$

to yield the dependence.

$\omega(k)=\omega_{0}+\varsigma / 2 \cdot\left(k-k_{0}\right)^{2}=\omega_{0}+\alpha\left(k-k_{0}\right)^{2}$, which is identical with dispersion relation (2) derived experimentally for localized plastic flow auto-waves.

Apparently, the dispersion relation of quadratic form $\omega(k)=\omega_{0}+\alpha\left(k-k_{0}\right)^{2}$, which is obtained for localized plastic flow auto-waves occurring at the stages of easy glide and linear work hardening, follows from the equality $\lambda \cdot V_{a w} \approx 1 / 2 d \cdot V_{\perp}$, which relates the macro-scopic characteristics of localized plastic flow auto-waves and the microscopic parameters of material crystal lattice.

\footnotetext{
${ }^{2}$ We had to overcome a certain difficulty since Dirac's hypothesis was initially applied to values in the ratio of about $10^{32}$
}

The right-hand side of Eq.3 can be rewritten as $1 / 2 d \cdot V_{\perp}=d^{2} \cdot \omega_{D}$ (here $\omega_{D}$ is the Debye frequency and $\left.V_{\perp} \approx 2 d \cdot \omega_{D}\right)$. Using the well-known formula $\hbar \omega_{D}=k_{B} \theta_{D}$ (here $k_{B}$ is the Boltzmann constant; $\hbar=h / 2 \pi$ is the Planck constant and $\theta_{D}$ is the temperature-ependent Debye parameter [22]), one can write

$$
1 / 2 d \cdot V_{\perp} \approx \lambda \cdot V_{a w} \approx d^{2} \cdot k_{B} \cdot \theta_{D}(T) / \hbar
$$

Eq.10 may be useful since it predicts the temperature dependence $\lambda V_{a w} \sim \theta_{D}(T)$ for localized plastic flow auto-waves [1].

\subsection{Group and Phase Rates of Localized Plastic Flow Autowaves}

In accordance with dispersion relation (2), the phase and group rates of localized plastic flow auto-waves can be represented in the dimensionless variables $\widetilde{\omega}$ and $\tilde{k}$ (see Figures 5(a) and 5(b)) as $\tilde{V}_{p h}=\widetilde{\omega} / \widetilde{k} \sim \tilde{k}+1 / \tilde{k}$ and $\tilde{V}_{g r}=d \widetilde{\omega} / d \tilde{k} \sim \tilde{k}$, respectively (see also Figure 3(a)).

From Eq.10 follows that

$$
V_{a w} \approx\left(d^{2} \cdot k_{B} \cdot \theta_{D} / \hbar\right) \cdot 1 / \lambda \approx\left(d^{2} \cdot k_{B} \cdot \theta_{D} / h\right) \cdot k \approx \xi \cdot k
$$

The experimental evidence suggests that $V_{g r} \sim k$. The quantity $\xi=d^{2} k_{B} \theta_{D} / h \approx d^{2} \cdot \omega_{D}$ from (11) is taken to be a proportionality coefficient, which can be readily calculated, using $d$ values reported in [15] and the Debye temperatures, $\theta_{D \mathrm{Fe}}=420 \mathrm{~K}$ and $\theta_{D \mathrm{Al}}=394 \mathrm{~K}$ obtained for the single crystals of iron and aluminum [22].

The calculated values of the proportionality coefficient obtained for the single crystals of iron and aluminum are, respectively, $\xi_{\mathrm{Fe}}=d^{2} \cdot k_{B} \theta_{D \mathrm{Fe}} / h \approx 3.7 \cdot 10^{-7}$ $\mathrm{m}^{2} \cdot \mathrm{s}^{-1}$ and $\xi_{\mathrm{Al}}=d^{2} \cdot k_{B} \theta_{D \mathrm{Al}} / h \approx 4.45 \cdot 10^{-7} \mathrm{~m}^{2} \cdot \mathrm{s}^{-1}$. The experimental values determined for the same materials from the inclination of $V_{g r}(k)$ plots are $\bar{\xi}_{\mathrm{Fe}}=(1 \pm$ $0.08) \cdot 10^{-7} \mathrm{~m}^{2} \cdot \mathrm{s}^{-1}$ and $\bar{\xi}_{\mathrm{Al}}=(12.9 \pm 0.15) \cdot 10^{-7} \mathrm{~m}^{2} \cdot \mathrm{s}^{-1}$, respectively. Matching of the calculated and the experimental values reveals a satisfactory agreement between the two sets of data.

It also follows from Figures 5(a) and 5(b) that the functions $\tilde{V}_{p h}(\tilde{k})$ and $\widetilde{V}_{g r}(\tilde{k})$ show fundamentally different behaviours for the stages of easy glide and linear work hardening: in the former case, they would not intersect for any $\widetilde{k}$ values, while in the latter case, they fully coincide for $\tilde{k} \geq 1$. The above difference in the function behaviours is attributable to the fact that the stage of easy glide is generally characterized by plastic 
flow instabilities, while at the stage of linear work hardening the plastic flow will occur in a steady-state regime, with the latter case evidently corresponding to the absence of dispersion, i.e. $\widetilde{\mathrm{V}}_{\mathrm{gr}}=\widetilde{\mathrm{V}}_{\mathrm{ph}}$.

\subsection{Change in the Entropy of the Deforming System by Auto-Wave Generation}

The dependencies $V_{w}(\theta)$ obtained for the above two types of wave are found to differ radically in form, i.e. $V_{p w} \sim \varepsilon^{1 / 2}$ and $V_{a w} \sim \theta^{-1}$. It might be also expected that the thermodynamic properties of the medium, in particular, entropy, will change in a dissimilar way by the generation of the two types of wave. It would appear reasonable to suggest that plastic deformation $\dot{\varepsilon} \sim V_{w}$ (here $V_{w}$ is the rate of a certain type of wave). Provided mobile dislocation density $\rho_{m} \approx$ const, the TaylorOrowan equation for plastic flow rate $\dot{\varepsilon}=b \rho_{m} V_{d i s l}$ can be applied to give $V_{\text {disl }} \sim \dot{\varepsilon} \sim V_{w}$. For thermally activated dislocation motion, the rate is given as $V_{\text {disl }} \sim \exp \left(-G / k_{B} \cdot T\right)$ (here $G=U-T S+A$ is the Gibbs thermodynamic potential; $\mathrm{U}$ is the internal energy; $\mathrm{S}$ is the entropy of the process; $A=-\gamma \sigma$ is the work of external stresses by the deformation and $\gamma$ is the activation volume of an elementary deformation act [23]). Hence the propagation rate is given for any type of wave as

$$
V_{w} \sim \dot{\varepsilon} \sim V_{d i s l} \sim \exp \left(S / k_{B}\right) \exp \left(-(U-\gamma \cdot \sigma) / k_{B} \cdot T\right)
$$

The enthalpy $H=U-\gamma \sigma$ observed for linear work hardening stage is virtually the same for most metallic materials. Consequently, taking the logarithm of (12) evidently yields $\ln V_{w} \sim S$.

Thus, a close correspondence is found to exist between the rectilinear diagrams $V_{w}(\theta)$ plotted in the co- ordinates $\ln V_{w}-\ln \theta$ for the both types of wave process (see Figure 6) on the one hand and the linear dependencies $S-\ln \theta$ obtained for the same processes on the other hand. The diagrams were plotted using wave rates listed in the table for the stage of easy glide in single crystals and for the stage of linear work hardening in single crystals and polycrystalline metals and alloys (Figure 6, lines 1 and 2, respectively). The wave rates were calculated from the expression $V_{p w} \approx\left(\theta / \rho_{0}\right)^{1 / 2}$ (Figure 6 , line 3 ) and the $\theta$ values, from the loading curves of investigated materials; besides, borrowed $\rho_{0}$ values were used [15].

An analysis of the dependencies $V_{w}(\theta)$ shows that in the case of plasticity waves, an increase in the entropy of the system would occur ( $\Delta S>0)$ (see Figure 6, line 3), which is characteristic for processes accompanied by dissipation of energy. In the case of auto-waves, the entropy of the system would decrease $(\Delta S<0$ ) (see Figure 6 , lines 1 and 2 for easy glide and linear work hardening, respectively).

The above results suggest that localized plastic flow waves differ radically from other types of wave process related to plastic deformation in solids. The generation of localized plasticity waves would cause a decrease in the entropy of the deforming system, which is an indication of its self-organization (ordering) [9] since entropy is a function of the parameter of order [24]. By considering localized plastic flow waves, the coefficient of work hardening $\theta<<1$ might be regarded as a parameter of order so that $S \sim \ln \theta$. With growing $\theta$ value, the entropy of the system would change linearly, with $\Delta S<0$ corresponding to auto-waves and $\Delta S>0$, to

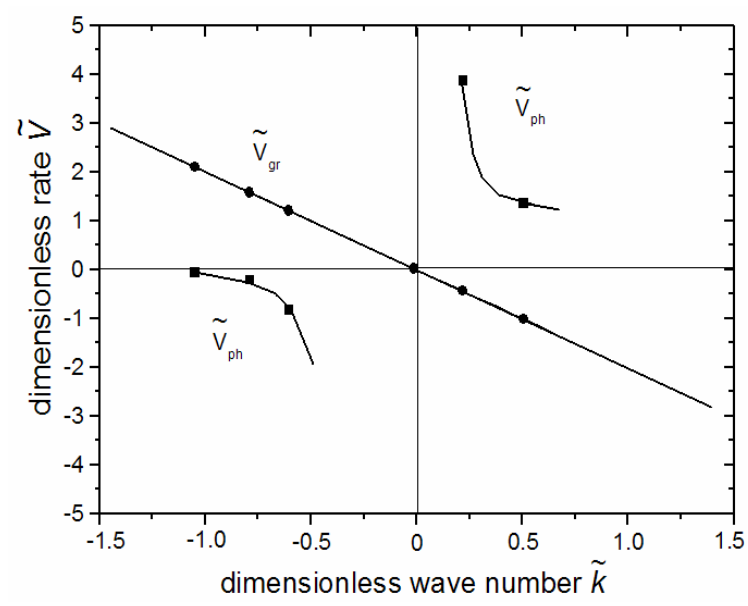

(a)

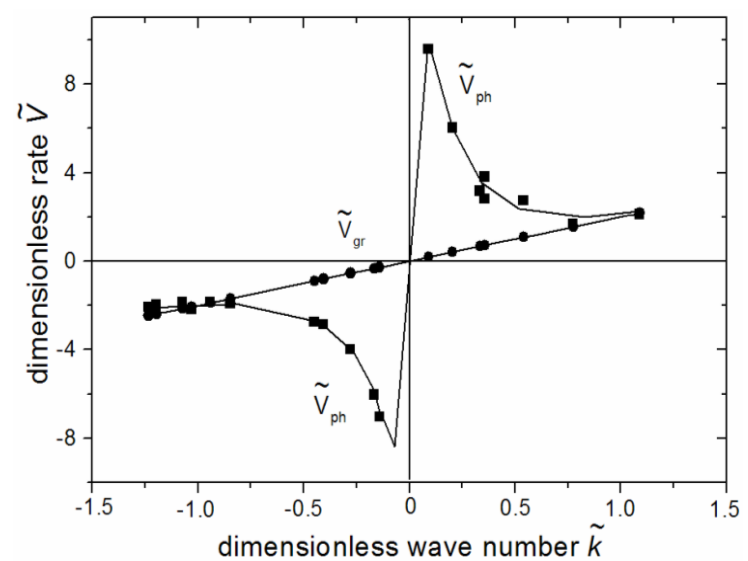

(b)

Figure 5. Wave number dependencies of phase ( $\bullet$ ) and group $(\bullet)$ propagation rates plotted for localized plastic flow auto-waves in the dimensionless invariables $\widetilde{V}-\widetilde{k}$; (a) easy glide stage; (b) linear work hardening stage. 


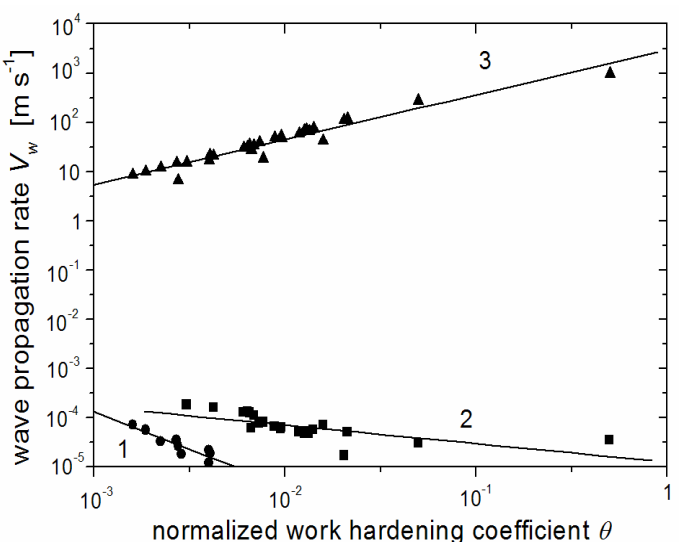

Figure 6. Changes in the entropies of plasticity waves (3) and of localized plastic flow auto-waves plotted for the stages of easy glide (1) and linear work hardening (2) in the co-ordinates $\ln V_{w}-\ln \theta$ (see the axis $\ln V_{w} \sim S$ ).

plasticity waves (see Figure 6).

The above suggests that plasticity waves [11] are commonly known dissipative processes, while localized plastic flow auto-waves are self-organization processes that are liable to cause a decrease in the entropy of the deforming system [9].

\subsection{Correspondence Between Localized Plastic Flow Patterns and Work Hardening Stages}

Of particular importance is the finding that localized plastic flow patterns emerging in a deforming solid are related to the respective flow stages [21]. These stages can be readily distinguished on the flow curve of the form [21]

$$
\sigma(\varepsilon)=\sigma_{0}+\theta \varepsilon^{n}
$$

where $\sigma_{0}$ is the proof stress and $n$ is the parabola exponent. The latter value will change discretely with the deformation, which enables individual stages to be singled out on the flow curve.

Using this method, a correspondence rule has been established for single crystals of metals and alloys and polycrystalline materials. This holds that

For $n=0$ (yield plateau) or $n \approx 0$ (easy glide), a solitary nucleus of localized plastic flow travels along the extension axis;

For $n=1$ (linear work hardening), localized plastic flow auto-waves are generated, which have wavelength and propagation rate $V_{a w}$;

For $n=1 / 2$ (parabolic work hardening or Tailor's stage), a set of immobile localized plastic flow nuclei is observed;

For $0<n<1 / 2$ (pre-failure stage), collapse of autowave takes place, which corresponds to macro-necking
[25] and

For $n=0$, ductile failure of material will occur.

The proposed rule evidently states that typical localization patterns observed on the macro-scale level are reflections of vastly different microscopic mechanisms involved in material work hardening at the different flow stages. This also testifies to the fact that the events involved in the deformation on the micro-scale level are directly related to those occurring on the macro-scale level in the deforming medium.

\section{CONCLUSIONS}

At all its stages the plastic flow involves localization processes that take the form of different types of autowave. The plastic flow tends to localize over the entire process; therefore, localization is taken to be its integral attribute.

The parameters of localized plastic flow evolution are found to be related to those of elastic deformation processes as $\lambda \cdot V_{a w} \approx 1 / 2 d \cdot V_{\perp}$. This suggests that the deformation process will exhibit scale invariance on both the micro-scale level $\left(d \cdot V_{\perp}\right)$ and the macro-scale one $\left(\lambda \cdot V_{a w}\right)$.

Due to the deformation process exhibiting invariance, the dispersion relation derived for localized plastic flow auto-waves that are generated at the stages of easy glide and linear work hardening has quadratic form, i.e. $\widetilde{\omega}=1 \pm \widetilde{k}^{2}$

A decrease in the entropy of the deforming medium strongly suggests that localized plasticity auto-waves are processes involved in the self-organization of the medium.

The localized plastic flow patterns are found to strictly correspond to the respective flow stages in single crystals and polycrystalline materials.

\section{AKNOWLEDGEMENTS}

This work was partly supported by the grant of RFBR (09-0800213-a).

\section{REFERENCES}

[1] Zuev, L.B. (2001) Wave phenomena in low-rate plastic flow of solids. Annals of Physics, 10(11-12), 965-984.

[2] Zuev, L.B. (2007) On the waves of plastic flow localization in pure metals and alloys. Annals of Physics, 16(1), 286-310.

[3] Zuev, L.B. and Danilov, V.I. (1999) A self-excited wave model of plastic deformation in solids. Philosophical Magazine A, 79(1), 43-57.

[4] Zuev, L.B. and Semukhin, B.S. (2002) Some acoustic 
properties of a deforming medium. Philosophical Magazine A, 82(6), 1183-1193.

[5] Zemskov, V.P. and Loskutov, A.Y. (2008) Oscillatory traveling waves in excitable media. Journal of Experimental and Theoretical Physics, 107(2), 344-349.

[6] Aifantis, E.C. (1996) Nonlinearly, periodicity and patterning in plasticity and fracture. International Journal of Non-Linear Mechanism, 31(8), 797-809.

[7] Aifantis, E.C. (1995) Pattern formation in plasticity. International Journal of Engineering Science, 33(15), 2161-2178.

[8] Aifantis, E.C. (2001) Gradient plasticity. In Lemaitre, J., Ed., Handbook of Materials Behavior Models, Academic Press, New York, 291-307.

[9] Haken, H. (1988) Information and self-organization. $A$ Macroscopic Approach to Complex Systems. Springer Verlag, Berlin.

[10] Zuev, L.B., Gorbatenko, V.V. and Polyakov, S.N. (2002) Instrumentation for speckle interferometry and techniques for investigating deformation and fracture. Proceedings of SPIE, 4900, 1197-1208.

[11] Kolsky, H. (1963) Stress waves in solid. Dover, New York.

[12] Barannikova, S.A. (2004) Dispersion of the plastic strain localization waves. Technical Physics Letters, 30(4), 338-340.

[13] Scott, A. (2003) Nonlinear science. University Press, Oxford.

[14] Braun, O.M. and Kivshar, Y.S. (2004) The FrenkelKontorova model. Springer, Berlin.

[15] Donnay, J.D.H. (1963) Crystal data. Determinate Tables,
Williams and Heintz Map Corp., Washington.

[16] Anderson, O.L. (1965) Determination and some applications of the isotropic elastic constants of polycrystalline systems, obtained from single crystals data. In Mason, W. P., Ed., Physical Acoustics: Principles and Methods, 3B, Academic Press, New York, 43-95.

[17] Dirac, P.A.M. (1978) Directions in physics. John Wiley and Sons, New York.

[18] Al'shits, V.I. and Indenbom, V.L. (1975) Phonon and electron drag of dislocations. Soviet Physics-Uspekhi, 115(18), 3-39.

[19] Nowick, A.S. and Berry, B.S. (1972) Anelastic relaxation in crystalline solids. Academic Press, New York.

[20] Kadomtsev, B.B. (1994) Dynamics and information. Physics-Uspekhi, 164(7), 449-530.

[21] Seeger, A. (1957) Mechanism of glide and work-hardening in face-centered cubic and hexagonal close packed metals. In Fisher, J.C., Ed., Dislocations and Mechanical Properties of Crystals, John Wiley and Sons, Inc., New York, 243-329.

[22] Ashcroft, N. and Mermin, N. (1976) Solid state physics. Holt, Rinehart and Winston, New York.

[23] Aifantis, E.C. and Gerberich W.W. (1975) A theoretical review of stress relaxation testing. Materials Science and Engineering, 21(4), 107-113.

[24] White, R.M. and Geballe, T.H. (1979) Long range order in solids. Academic Press, New York.

[25] Zuev, L.B., Danilov, V.I. and Barannikova, S.A. (2008) Plastic flow, necking and failure in metals, alloys and ceramics. Materials Science and Engineering A, 483-484, 223-227. 